\title{
ON THE DETERMINATION OF THE PHASE OF A FOURIER INTEGRAL, II ${ }^{1}$
}

\section{EDWIN J. AKUTOWICZ}

1. Introduction. In the present note we make a further remark upon the problem of finding sufficient conditions for a complexvalued function $\phi(t),-\infty<t<\infty$, which together with the modulus of its Fourier transform determine it to within specifiable transformations. In an earlier paper [1] on this subject we have found that if $\mathfrak{C}(a)$ is the class of all complex-valued functions $\phi$ such that

(A) $\phi \in L^{1}(-\infty, \infty) \cap L^{2}(-\infty, \infty)$,

(B) $\phi$ is equivalent to zero on some half-line, $t<t_{0} \equiv t_{0}(\phi)$,

(C) $\widehat{\phi}(x) \neq 0,-\infty<x<\infty$, where $\widehat{\phi}$ denotes the Fourier transform of $\phi$,

(D) $|\widehat{\phi}(x)|$ is equal to a given function, $a(x),-\infty<x<\infty$, then any two functions $\phi_{1}, \phi_{2}$ belonging to $\mathfrak{e}(a)$ are related by an equation of the form:

$$
B_{2}(x) \hat{\phi}_{1}(x)=e^{i \alpha+i \beta x} B_{1}(x) \hat{\phi}_{2}(x), \quad-\infty<x<\infty,
$$

where $\alpha, \beta$ are real numbers and $B_{1}(x+i y), B_{2}(x+i y)$ are analytic functions for $y \geq 0$ of modulus identically 1 on $y=0$, which are given as certain Blaschke products. $B_{k}(z)$ has as zeros the set of zeros $\left\{z_{n k}\right\}$ of the function

$$
\Phi_{k}(z)=\frac{1}{(2 \pi)^{1 / 2}} \int_{t_{0}}^{\infty} e^{i z t} \phi_{k}(t) d t, \quad(z=x+i y, y>0, k=1,2)
$$

and is given by the product:

$$
B_{k}(z)=\left(\frac{z-i}{z+i}\right)^{m_{k}} \prod_{n} \frac{\left|z_{n k}-i\right|}{z_{n k}-i} \cdot \frac{\left|z_{n k}+i\right|}{z_{n k}+i} \cdot \frac{z-z_{n k}}{z-\bar{z}_{n k}}
$$

where $m_{k}$ is a non-negative integer. The necessary and sufficient condition that (1.3) converge is that

$$
\sum_{n} \operatorname{Im} z_{n k} /\left(1+\left|z_{n k}\right|^{2}\right)<+\infty .
$$

What is investigated in the sequel is the degree to which the non-

Presented to the Society, October 27, 1956; received by the editors May 25, 1956.

1 The research in this document was supported jointly by the Army, Navy, and Air Force under contract with the Massachusetts Institute of Technology, Lincoln Laboratory. 
uniqueness of phase, expressed in (1.1), is decreased if assumption (B) is replaced by the stronger condition:

$\left(\mathrm{B}^{1}\right) \phi$ is equivalent to zero outside a finite interval, which may a priori depend upon $\phi$.

It will be observed that in (1.1) there appear (the limits of) two Blaschke products which are defined in terms of their respective zeros, $\left\{z_{n 1}\right\}$ and $\left\{z_{n 2}\right\}$. In the circumstances (A), (B), (C), (D) these sequences are necessarily without finite limit points but are otherwise more or less arbitrary (subject, of course, to the convergence of the products). In particular, any finite number of zeros can be added and subtracted. In the circumstances $(A),\left(B^{1}\right),(C),(D)$ these two previously largely independent sequences of zeros reduce to $\left\{z_{n 1}\right\}$ and $\left\{\bar{z}_{n 1}\right\}$. Thus one can state, somewhat sententiously, that the restriction $\left(\mathrm{B}^{1}\right)$ being twice as severe as $(\mathrm{B})$ results in a reduction of leeway in the phases by fifty per cent.

I am indebted to R. C. T. Smith for criticizing earlier attempts of mine on the problem at hand.

2. Proof of the reduction. Let $\mathfrak{C}_{0}(a)$ denote the class of all complexvalued functions satisfying (A), ( $\left.\mathrm{B}^{1}\right),(\mathrm{C}),(\mathrm{D})$. Then $\mathfrak{e}_{0}(a) \subset \mathfrak{e}(a)$. Let $\phi \in \mathfrak{C}_{0}(a)$, and suppose $(-T, T)$ is the smallest interval centered at the origin outside of which $\phi=0$ almost everywhere. Put

$$
\Phi(z)=\frac{1}{(2 \pi)^{1 / 2}} \int_{-T}^{T} e^{i z t} \phi(t) d t, \quad z=x+i y .
$$

Then by $\S 3$ of $[1]$ we can write

$$
\Phi(z)=e^{i \alpha+i \beta_{z}} B(z) D(z),
$$

where $\alpha, \beta$ are real, $B(z)$ is the Blaschke product in the upper halfplane formed with the zeros of $\Phi$ which lie in the upper half-plane, and

$$
D(z)=\exp \left(\frac{1}{\pi i} \int_{-\infty}^{\infty} \frac{1+u z}{u-z} \frac{\log a(u)}{1+u^{2}} d u\right) .
$$

Since $\Phi(z)$ is an entire function and satisfies

$$
\Phi(z)=O\left(e^{T|z|}\right),
$$

we also have

$$
\Phi(z)=e^{c(z)} P(z),
$$

where $c(z)=c_{0}+c_{1} z$ for some complex constants $c_{0}, c_{1}$, and $P(z)$ is the canonical product formed with all the zeros of $\Phi$. $P(z)$ has genus 0 
or 1 , (see $[2$, p. 250]). We shall assume the genus to be 1 , as the same argument, slightly simplified, can be applied for genus 0 .

Suppose now that $\phi_{1}$ and $\phi_{2}$ both belong to $\mathcal{C}_{0}(a)$, and let $\Phi_{1}, \Phi_{2}$ be given by (2.1) in terms of $\phi_{1}, \phi_{2}$, respectively. Since $D(z)$ depends only upon $a,(2.2)$ implies

$$
B_{2}(z) \Phi_{1}(z)=e^{i \alpha^{\prime}+i \beta^{\prime} s} B_{1}(z) \Phi_{2}(z),
$$$$
(y>0) \text {, }
$$

where $B_{1}, B_{2}$ are the Blaschke products in the upper half-plane associated with $\Phi_{1}, \Phi_{2}$, and $\alpha^{\prime}, \beta^{\prime}$ are real. By (2.4) and (2.5)

$$
B_{2}(z) P_{1}(z)=e^{m(z)} B_{1}(z) P_{2}(z), \quad(y>0),
$$

where

$$
P_{1}(z)=\prod_{n}\left(1-\left(z / z_{n}\right)\right) e^{z / z_{n}}, \quad P_{2}(z)=\prod_{n}\left(1-\left(z / \zeta_{n}\right)\right) e^{z / \zeta_{n}},
$$

and $m(z)=m_{0}+m_{1} z .\left\{z_{n}\right\}$ is the set of all zeros of $\Phi_{1}$, and $\left\{\zeta_{n}\right\}$ that of $\Phi_{2}$, both sets being enumerated according to increasing magnitude. We can assume that $\left\{z_{n}\right\}$ and $\left\{\zeta_{n}\right\}$ are disjoint, as any common zeros can be cancelled in (2.6).

Writing $z_{n}=x_{n}+i y_{n}, y_{n}>0$, and taking $0<K<1$, we have for all sufficiently large $n$ :

$$
\left|\log \left\{\left(1-\left(z / \bar{z}_{n}\right)\right) e^{z / z_{n}}\right\}\right|<2|z| \cdot \frac{y_{n}}{\left|z_{n}\right|^{2}}+\frac{|z|^{2}}{1-K} \cdot \frac{1}{\left|z_{n}\right|^{2}},
$$

where the branch of the logarithmic function appearing is that which is zero for $z=0$. By (1.4), together with the fact that $\left|z_{n}\right| \rightarrow \infty$ as $n \rightarrow \infty$, we have $\sum_{y_{n}>0} y_{n} /\left|z_{n}\right|^{2}<+\infty$. Also $\sum_{n} 1 /\left|z_{n}\right|^{2}<+\infty$, since the genus is 1 . Hence

$$
\prod_{\operatorname{Im} z_{n}>0}\left(1-\left(z / \bar{z}_{n}\right)\right) e^{z / z_{n}}
$$

is a convergent product in the entire $z$-plane. The same is true of

$$
\prod_{\operatorname{Im} \zeta_{n}>0}\left(1-\left(z / \bar{\zeta}_{n}\right)\right) e^{z / \zeta_{n}}
$$

Equation (2.6) can be written for $y>0$ in the form

$$
\begin{aligned}
& \prod_{\operatorname{Im} z_{n}<0}\left(1-\left(z / z_{n}\right)\right) e^{z / z_{n}} \\
= & e^{m(z)} \prod_{\operatorname{Im} z_{n}>0, \operatorname{Im} \zeta_{n}>0} \gamma_{n} \cdot \frac{z_{n} \bar{\zeta}_{n}\left(1-\left(z / \bar{\zeta}_{n}\right)\right) e^{z / \zeta_{n}}}{\bar{z}_{n} \zeta_{n}\left(1-\left(z / \bar{z}_{n}\right)\right) e^{z / z_{n}}} \cdot \prod_{\operatorname{Im} \zeta_{n}<0}\left(1-\left(z / \zeta_{n}\right)\right) e^{z / \zeta_{n}},
\end{aligned}
$$

where $\gamma_{n}$ is a constant of absolute value 1 arising from the Blaschke 
products (see (1.3)). Since the products (2.7) and (2.8) are convergent, it follows that

$$
\prod_{\operatorname{Im} z_{n}>0, \operatorname{Im} \zeta_{n}>0} \gamma_{n} z_{n} \bar{\zeta}_{n} / z_{n} \zeta_{n}
$$

is convergent to a limit, which must be of absolute value 1 . Absorbing this into $e^{m(z)},(2.9)$ can be put in the form

$$
\begin{aligned}
\prod_{\operatorname{Im}} & \left(1-\left(z / z_{n}<0\right) e^{z / z_{n}} / \prod_{\operatorname{Im} \zeta_{n}>0}\left(1-\left(z / \bar{\zeta}_{n}\right)\right) e^{z / \zeta_{n}}\right. \\
= & e^{m(z)} \prod_{\operatorname{Im} \zeta_{n}<0}\left(1-\left(z / \zeta_{n}\right)\right) e^{z / \zeta_{n}} / \prod_{\operatorname{Im} z_{n}>0}\left(1-\left(z / \bar{z}_{n}\right)\right) e^{z / z_{n}},
\end{aligned}
$$

and (2.10) is valid for all finite $z$. Hence the zeros and poles must be the same on both sides of (2.10). But as $\left\{z_{n}\right\}$ and $\left\{\zeta_{n}\right\}$ are disjoint this is possible only if none exist. Therefore $\left\{z_{n}\right\}=\left\{\bar{\zeta}_{n}\right\}$. If we put $\left\{z_{n}\right\}=\left\{z_{n}^{\prime}\right\} \cup\left\{z_{n}^{\prime \prime}\right\}$, where the $z_{n}^{\prime}$ lie in the upper half-plane and the $z_{n}^{\prime \prime}$ in the lower half-plane, (1.1) can be written

$$
\widehat{\phi}_{1}(x)=e^{i \alpha+i \beta x} B_{2}^{*}(x) B_{1}(x) \widehat{\phi}_{2}(x),
$$

where

$$
B_{2}^{*}(x)=\lim _{\nu \rightarrow 0} \prod_{\left\{z_{n}^{\prime \prime}\right\}} \frac{\left|z_{n}^{\prime \prime}-i\right|}{z_{n}^{\prime \prime}-i} \cdot \frac{\left|z_{n}^{\prime \prime}+i\right|}{z_{n}^{\prime \prime}+i} \frac{z-z_{n}^{\prime \prime}}{z-\bar{z}_{n}^{\prime \prime}} .
$$

We can summarize our results in the following

TheOREm. Let $\mathfrak{C}_{0}(a)$ be the class of all complex-valued functions $\phi$ satisfying the following conditions:

(i) ${ }^{2} \phi \in L^{2}(-\infty, \infty)$,

(ii) $\phi$ vanishes almost everywhere outside a finite interval which may depend upon $\phi$,

(iii) $\widehat{\phi}(x) \neq 0,-\infty<x<\infty$,

(iv) $|\widehat{\phi}(x)|$ is equal to a fixed function, $a(x)$. Then any two functions $\phi_{1}, \phi_{2}$ belonging to $\mathfrak{C}_{0}(a)$ are related by an equation of the form (2.11.)

The argument used to derive this result does not appear to be applicable if the hypotheses are significantly weakened within $L^{2}(-\infty$, $\infty)$. Thus although $\Phi$ may be entire without $\phi$ vanishing a.e. outside a finite interval, this latter circumstance is necessary if $\phi$ belongs to $L^{2}(-\infty, \infty)$ and $\Phi$ is of exponential type, on account of the PaleyWiener theorem. Also, the canonical representation (2.2) upon which the procedure rests is strongly dependent upon assumption (iii).

${ }^{2}$ Condition (i) is equivalent to (A) in the presence of (ii). 
3. An illustration. Finally, we show by an example that the above theorem is the best possible in the sense that a sharper determination of phase is out of the question, even if the conditions in $\$ 2$ are strengthened by requiring that $\phi$ be non-negative.

Consider the functions

$$
\phi_{1}(t)= \begin{cases}e^{-t} & \text { if }-1 \leq t \leq 1 \\ 0 & \text { otherwise }\end{cases}
$$

and

$$
\phi_{2}(t)= \begin{cases}e^{t} & \text { if }-1 \leq t \leq 1 \\ 0 & \text { otherwise }\end{cases}
$$

Then

$$
\widehat{\phi}_{1}(x)=\left(\frac{2}{\pi}\right)^{1 / 2} \frac{1}{i x-1} \sinh (i x-1),
$$

and

$$
\widehat{\phi}_{2}(x)=\left(\frac{2}{\pi}\right)^{1 / 2} \frac{1}{i x+1} \sinh (i x+1),
$$

so that $\left|\hat{\phi}_{1}(x)\right|=\left|\hat{\phi}_{2}(x)\right|$, and the zeros associated with $\phi_{1}$ are

$$
z_{n}=-i+n \pi, \quad n= \pm 1, \pm 2, \cdots,
$$

and those with $\phi_{2}$ are

$$
\zeta_{n}=i+n \pi, \quad n= \pm 1, \pm 2, \cdots .
$$

\section{REFERENCES}

1. E. J. Akutowicz, On the determination of the phase of a Fourier integral, I., Trans. Amer. Math. Soc. vol. 83 (1956) pp. 179-192.

2. E. C. Titchmarsh, The theory of functions, 2d ed., Oxford, 1939.

Massachusetts Institute of Technology 